THE INTERNATIONAL

REVIEW OF RESEARCH IN

OPEN AND DISTANCE LEARNING

\title{
An E-Book Hub Service Based on a Cloud Platform
}
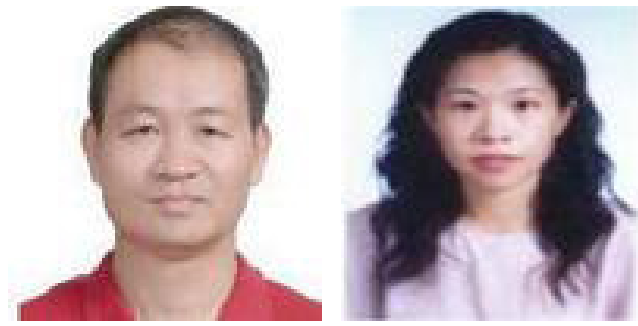

Jinn-Shing Cheng and Echo Huang National Kaohsiung First University of Science and Technology, Taiwan, Republic of China Chuan-Lang Lin Hamastar Technology Co., Taiwan, Republic of China

\section{Abstract}

Due to the constant performance upgrades and regular price reductions of mobile devices in recent years, users are able to take advantage of the various devices to obtain digital content regardless of the limitations of time and place. The increasing use of e-books has stimulated new e-learning approaches. This research project developed an e-book hub service on a cloud computing platform in order to overcome the limitations of computing capability and storage capacity that are inherent in many mobile devices. The e-book hub service also allows users to automatically adjust the rendering of multimedia pages at different resolutions on terminal units such as smartphones, tablets, PCs, and so forth. We implemented an e-book hub service on OpenStack, which is a free and open-source cloud computing platform supported by multiple large firms. The OpenStack platform provides a large-scale distributed computing environment that allows users to build their own cloud systems in a public, private, or hybrid environment. Our e-book hub system offers content providers an easy-to-use cloud computing service with unlimited storage capacity, fluent playback, high usability and scalability, and high security characteristics to produce, convert, and manage their e-books. The integration of information and communication technologies has led the traditional publishing industry to new horizons with abundant digital content publications. Results from this study may help content providers create a new service model with increased profitability and enable mobile device users to easily get digital content, thereby achieving the goal of e-learning.

Keywords: E-learning; cloud computing; e-book; e-book hub; e-reader; digital content 


\section{Introduction}

When an American company called SoftBook Press produced an e-reader called the SoftBook in 1998 (n.d.; Schilit, Golovchinsky, Tanaka, \& Marshall, 1999), the device wasn't popular because there were just a few suppliers of e-books and e-readers at the time. After Amazon released the Kindle in 2007 and Apple the first iPad in 2010 (Amazon Kindle, n.d.), the e-book business began to flourish, and digital content became a new trend in the related publishing and education industries (Chou, Jay, Lin, \& Hsieh, 2010; Shen \& Koch, 2011; Yee, Chia, Tsai, Tiong, \& Rajaraman, 2011; Rahman, Alam, \& Saddik, 2011). According to statistics from the Association of American Publishers (Sporkin, 2012), the total volume of e-book sales in the American market has exceeded physical books since January 2011, when volume increased $116 \%$ over January 2010. Compared to the sales volume in February 2010, the range of increase reached 202\%. Besides the e-reader, digital content providers, e-book transaction platforms, and network communication systems are needed to bolster the e-book market in order to create an e-book generation-one that is digital and paperless.

Recently, the number of users accessing e-book services has increased due to the latest improvements in the capability of cloud computing. Because of the storage limitations and computing capability inherent in many mobile devices (Haber, 1999; Sahu, Sharma, Dubey, \& Tripathi, 2012), more and more companies store e-books in the unlimited and low-priced space offered by cloud servers. However, there is currently no appropriate solution to solve the load balancing issue when a cloud computing facility is used to maintain e-book systems.

The purpose of our research was to design and build an e-book hub service with multimedia editing capabilities based on open-source resources of cloud computing technology. The service we implemented successfully allowed content providers to easily edit and convert their e-books and enabled them to manage e-book hub services in an unlimited space offered by the scalable, robust, and safe cloud computing environment.

\section{Cloud Platform Technology}

The cloud platform technology we used in this research project is an open-source platform called OpenStack (2012a), freeware supported by industry insiders. OpenStack is a cloud operating system that adopted the authority clause of Apache Licence 2.0, and it may operate as a private, public, or hybrid cloud environment. OpenStack's extendable and flexible cloud environment can coordinate with two main public cloud providers, Amazon and Rackspace, and support other cloud environments in the future.

The three main kernels of OpenStack are Compute, Object Storage, and Image Service. Figure 1 shows the system's architecture, and we have illustrated the system.

\section{Compute Infrastructure (Nova)}

OpenStack Compute (2012b), code-named Nova, is open-source software designed to pro- 
vision and manage large networks of virtual machines, creating a redundant and scalable cloud computing platform.

\section{Storage Infrastructure (Swift)}

OpenStack Object Storage (2012d), code-named Swift, is open-source software that creates redundant, scalable object storage using clusters of standardized servers to store petabytes of accessible data.

\section{Imaging Service (Glance)}

OpenStack Image Service (2012c), code-named Glance, provides discovery, registration, and delivery services for virtual disk images.

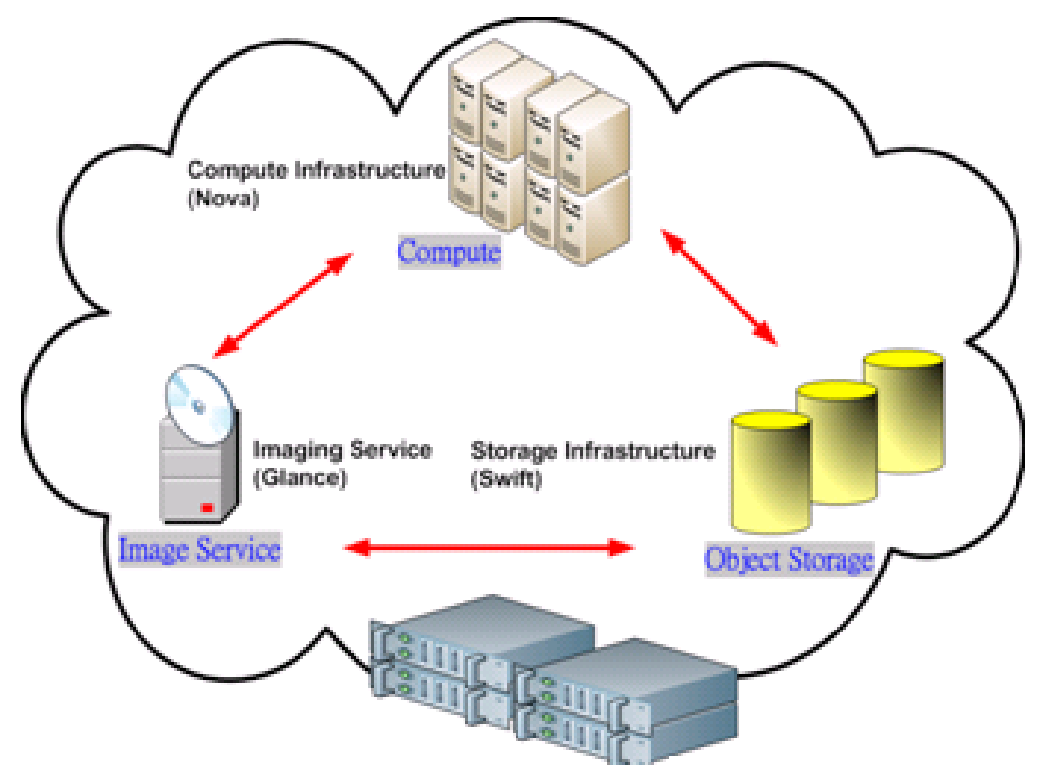

Figure 1. Architecture of OpenStack.

\section{The Components of the E-Book Hub System}

The architecture of the standalone client-server e-book hub system we developed in this research project is shown in Figure 2. We constructed the system with Microsoft IIS, .Net applications, and Microsoft SQL Server databases. Its components are divided into three parts: the access device, the physical network, and the APPs service. 


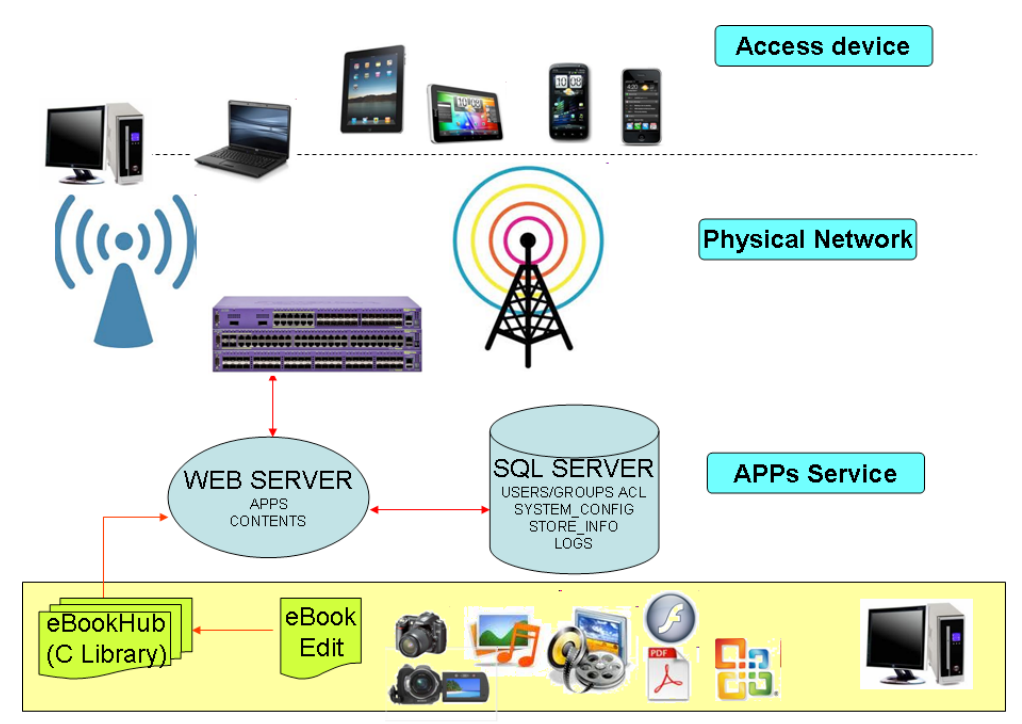

Figure 2. E-book system architecture.

\section{The E-Book Editor}

An e-book editor program called SimMAGIC eBook is user-friendly software for editing or converting word processor files into e-book formats. Users can use the editor to combine all the components-text, images, video, and audio-together to create their own e-books. The editor also allows users to transform supported files like PDF or PPT into e-books and then upload them to the e-book hub server. The development tools used to create the ebook editor included Visual Studio C\#, Xcode, Eclipse, and Silverlight, among others. Its functions allow a user to define, edit, preview, simulate, and export e-book files. Snapshots of the function, editing, and operating interfaces in SimMAGIC eBook are shown in Figures 3,4 , and 5 . 


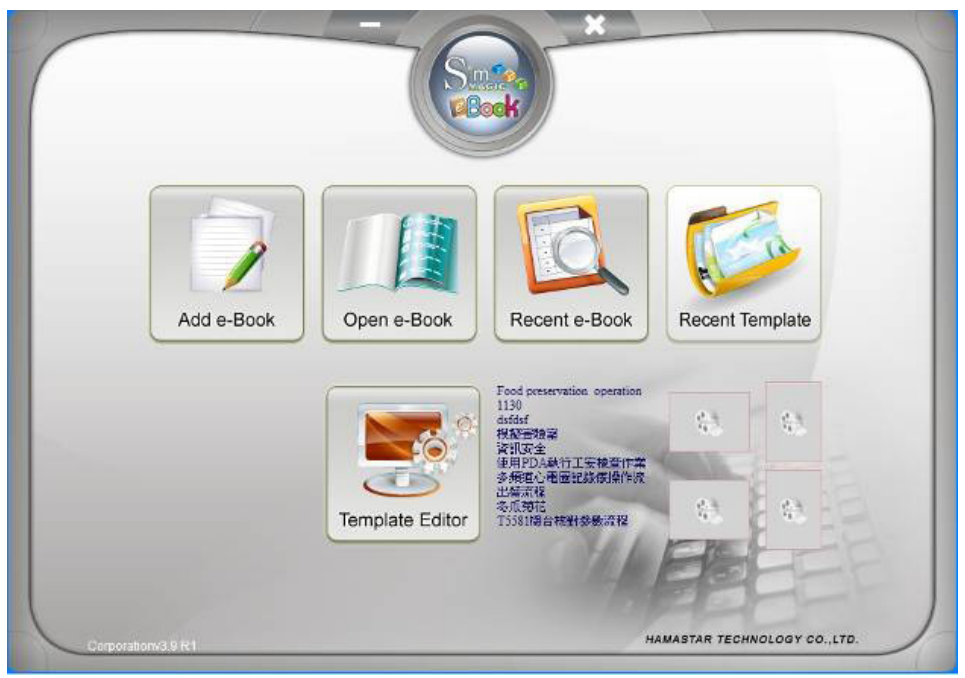

Figure 3. SimMAGIC ebook functions.

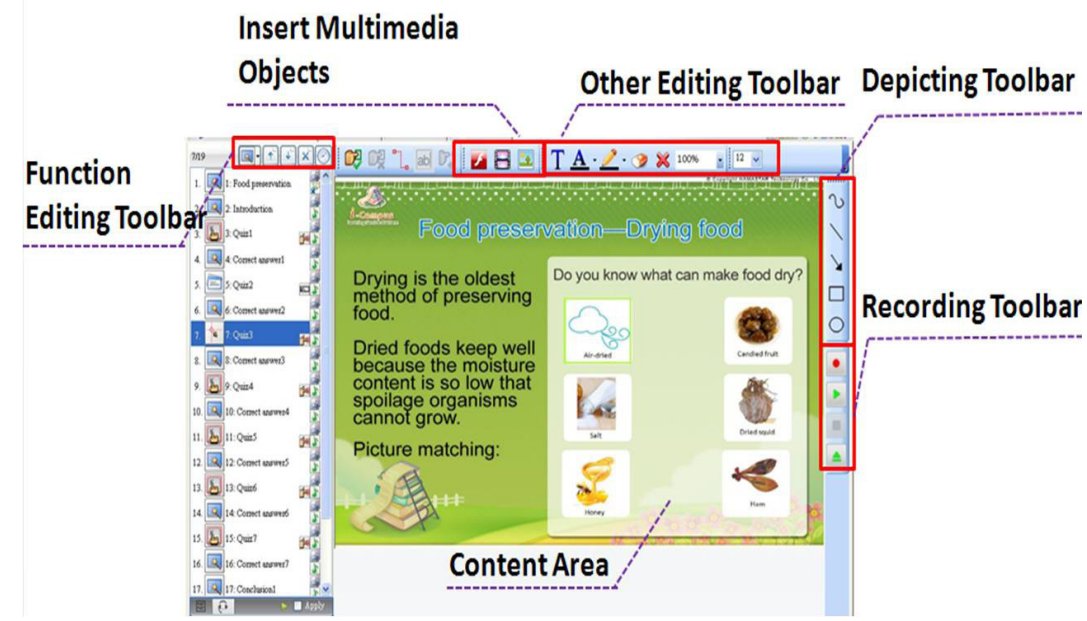

Figure 4. SimMAGIC e-book editing application layout.

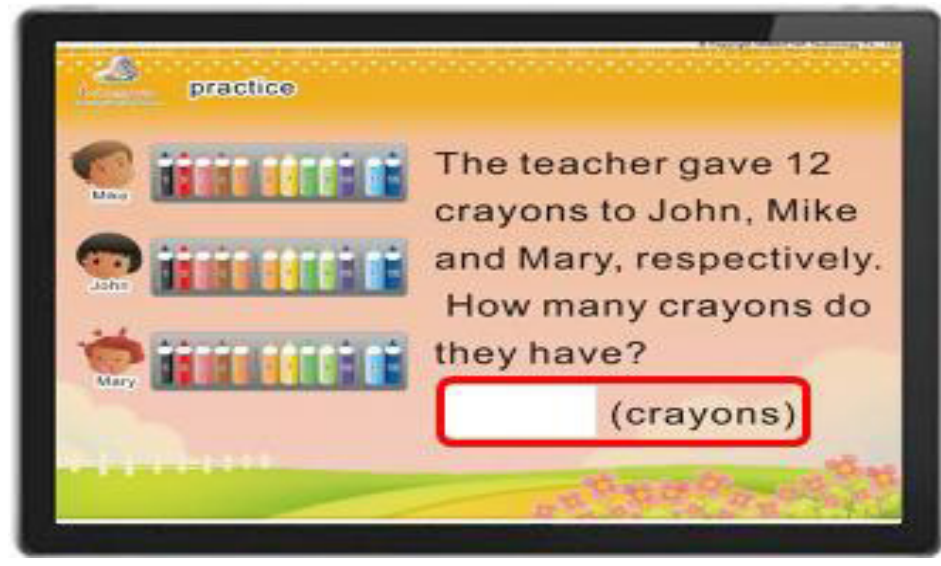

Figure 5. SimMAGIC e-book interactive operation. 


\section{Cloud Library}

The cloud library called SimMAGIC C-Library is a combination e-book hub web server, content server, and database server that enables users to store, manage, and provide e-book content service. The development tools used to create the C-Library included Visual Studio 2010, .Net Framework 4.o, ASP.Net C\#, JavaScript, HTML, and AJAX, among others. Its functions include the uploading and downloading of e-books, e-book hub classification and management, user registration and management, e-book catalogue searches, and statistical analysis. A snapshot of the web portal for the C-Library is illustrated in Figure 6.

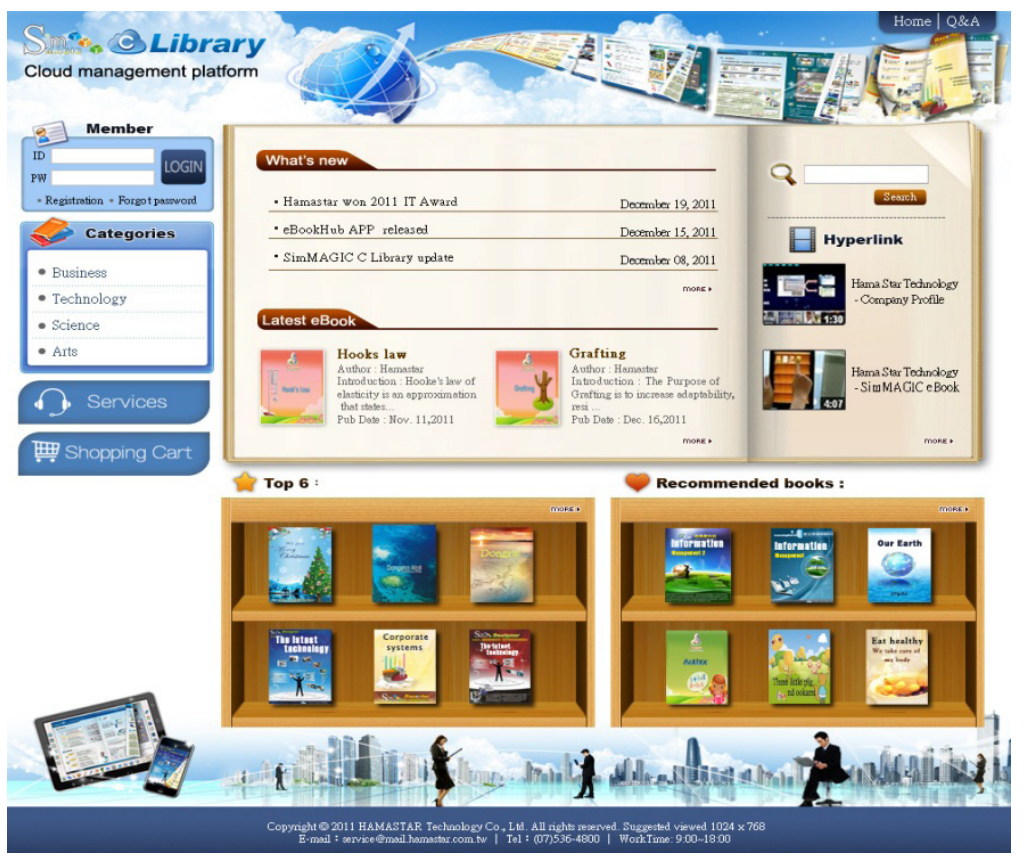

Figure 6. SimMAGIC C-Library portal.

\section{Mobile Device Applications}

The application E-book Hub can be used on any type of mobile device with an iOS, Android, or Windows operating system to upload or download e-books. Figures 7 and 8 show snapshots of the application's interface. 

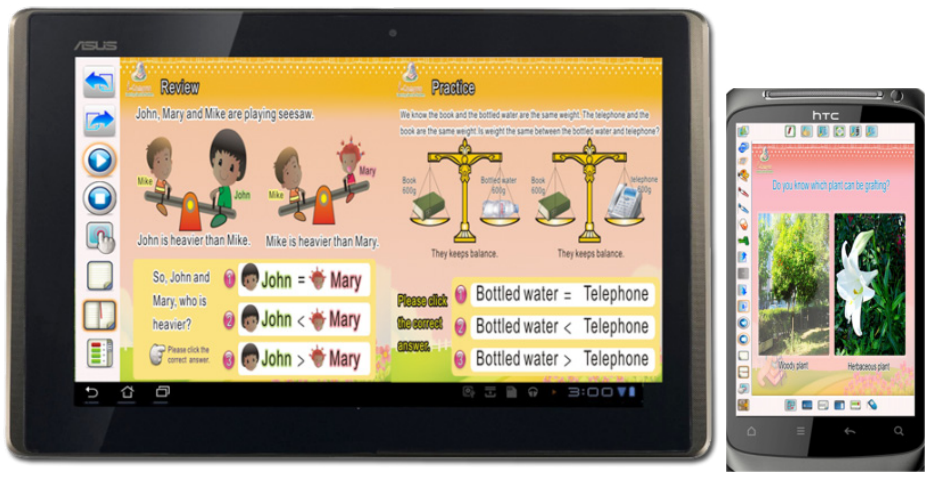

Figure 7. E-book Hub on a device with an Android operating system.

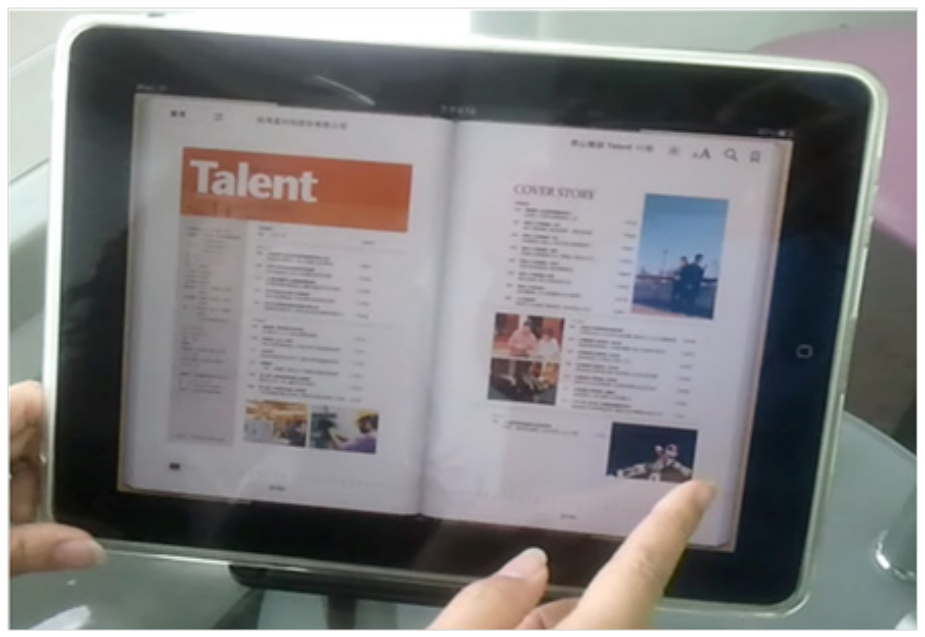

Figure 8. E-book Hub displaying an EPUB publication.

\section{Cloud E-Book Hub System}

The standalone e-book hub system we described in the last section has limitations in network traffic utilization, load balancing control, and resource management because it lacks an automatic adjustment control that would enable it to respond quickly to users' demands, and this problem makes it prohibitively expensive to set up the physical infrastructure and operating systems. This has limited the desire of companies to invest money in a standalone e-book hub system. Utilizing cloud computing technology with its high capability, fluency, extendibility, and security at a low cost may provide a solution to the problem.

To this end, we have designed and implemented a cloud technology-based e-book hub system in this study. To transform physical servers into a cloud platform, we used OpenStack, an open-source cloud platform in a private cloud environment, and it provided both infrastructure as a service (IaaS) and platform as a service (PaaS) utilities. We developed the cloud-based e-book hub system according to the framework depicted in Figure 9. We used OpenStack Compute Infrastructure to develop and manage the cloud e-book hub system, which included virtual servers for Web site hosting, content provision, multimedia edit- 
ing, data backup, network traffic control, and load monitoring. Then we utilized OpenStack Imaging Service to develop, manage, and store images on the servers. Finally, we employed OpenStack Storage Infrastructure to provide an environment in which to store all the servers, e-book content, and images.

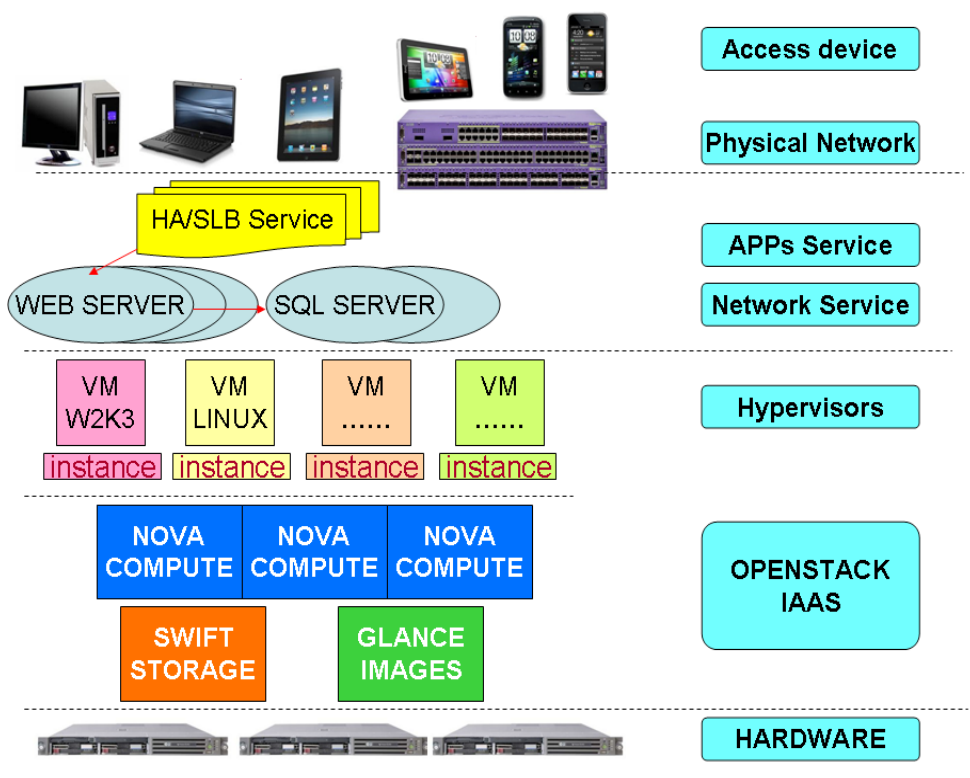

Figure 9. Our cloud e-book hub system framework.

The hardware for the whole system included physical servers, network infrastructure, and users' mobile devices. The physical servers used to build an OpenStack platform provided Cloud Control, Cloud Compute, Swift Storage, and Glance Image services.

\section{Cloud Control - Cloud Compute Service}

The Cloud Compute service controls the operation and resource management of a cloud system. It also constructs virtual machines in the environment, as depicted in Figure 10. 


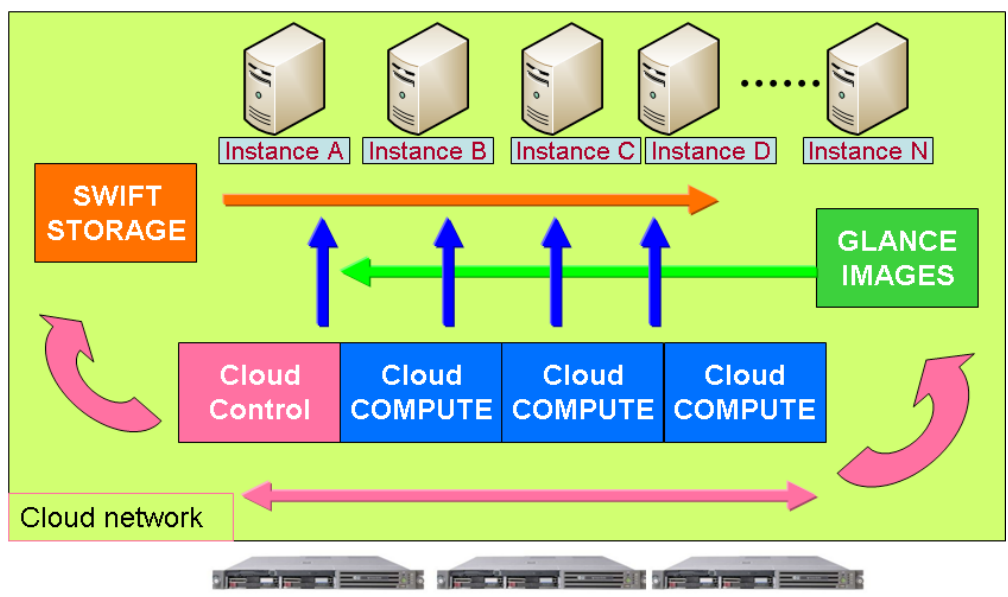

Figure 10. Cloud Control and Cloud Compute service.

\section{Swift Storage Service}

The Swift Storage service provides space for all the services in a cloud system. With the characteristics of unlimited storage, built-in replication, higher performance, and highly scalable read/write accessibility, the service enhances the reliability, completeness, and extendibility of the cloud system. The service is depicted in Figure 11.

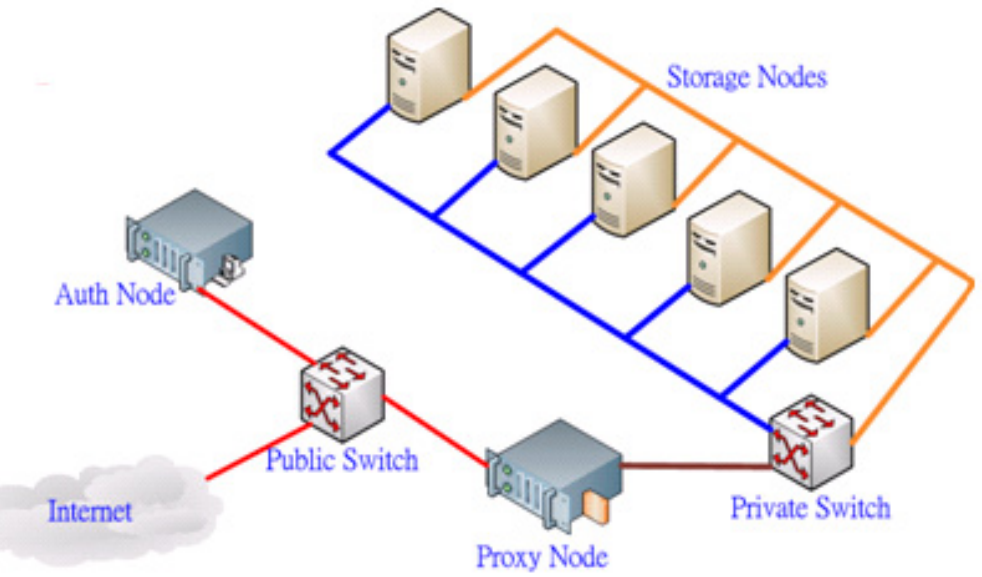

Figure 11. Swift Storage service. 


\section{Glance Image Service}

The Glance Image service manages virtual machines in the cloud environment. For example, images for virtual web servers may be automatically launched or shut down to accommodate heavier or lighter web requests. The service architecture is shown in Figure 12.

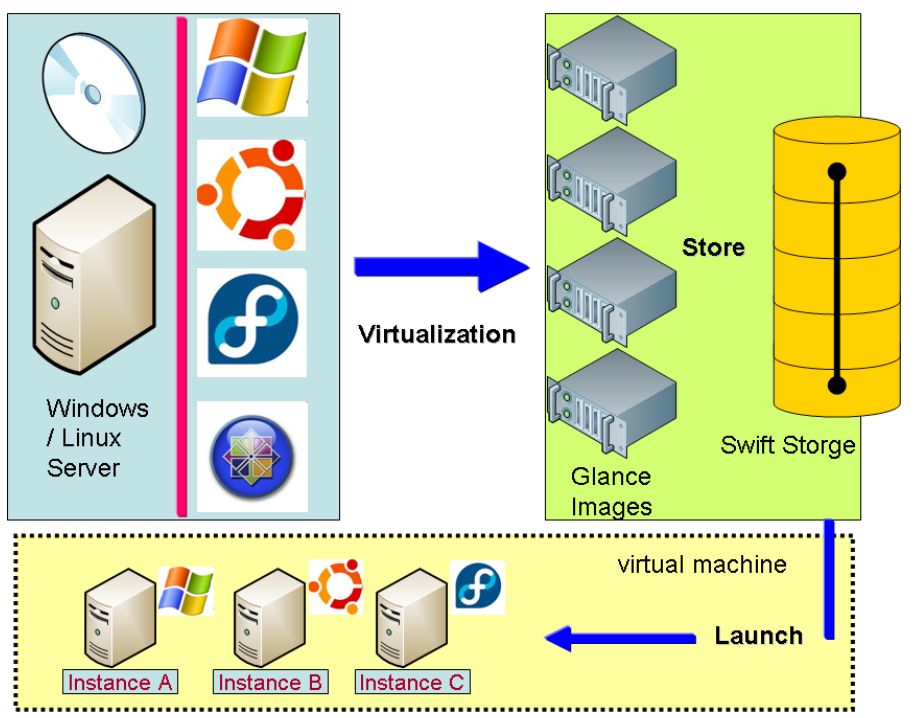

Figure 12. Glance Image service.

We used a high availability (HA)-server load balancing feature to provide high reliability, even flow distribution, and load balance for the virtual web servers. Our objective was to maintain the overall robustness of the system, so that when one of the cloud compute nodes was overloaded, the virtual web servers hosted on others continued working. Based on our desired strategy for traffic distribution, the system could use round-robin, least-connection, or weighted distribution algorithms to balance the traffic rate and decrease the chances of distributed denial of service (DDoS). In addition, SNMP and Windows management instrumentation (WMI) helped monitor the load and traffic rate on the server. With the automatic adjustment program, the Glance Image service increased or decreased instances of web servers according to predefined thresholds for loads and traffic. This automatic adjustment capability clearly demonstrated the advantage of cloud technology in resource management. All the virtual servers operated on instances constructed by cloud compute nodes, and their storage space was provided by the Swift Storage service. A framework for the entire cloud e-book system is depicted below. 


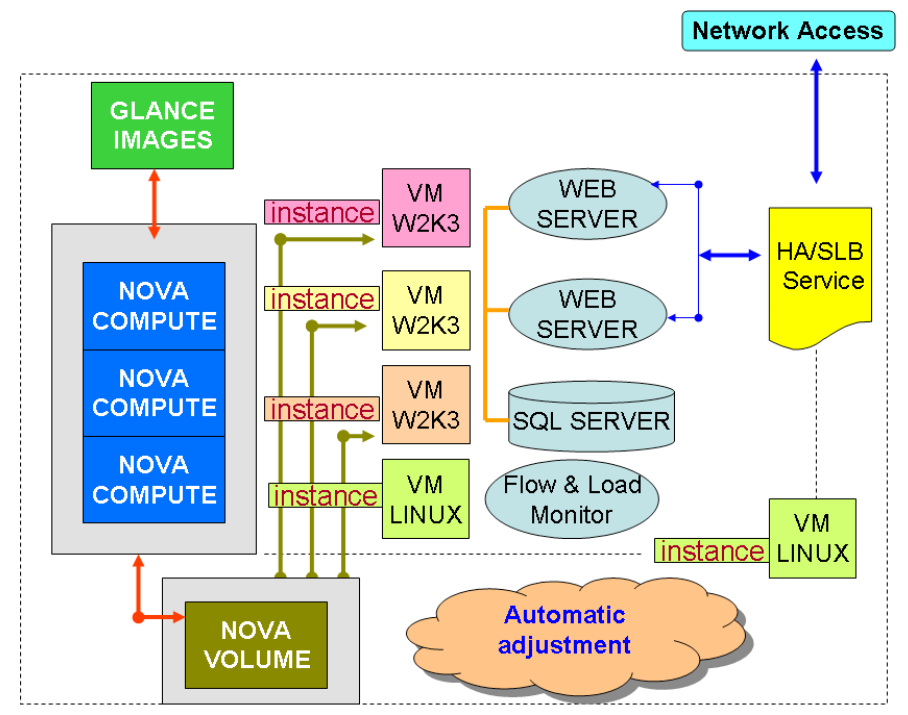

Figure 13. E-book cloud service.

\section{System Performance Verification}

We used the Apache Jmeter (2012), an open-source testing tool, to verify the performance of the systems we implemented in this study. The tool was used to measure the performance of a standalone e-book hub system and a cloud-based e-book hub system equipped with HA-server load balancing, as illustrated in Figure 13.

The elements that made up the test environment are shown in Table 1. To create a test environment close to the application's real-life situation, we set up cloud services in the server farm of a school and used Apache Jmeter to test the system's performance from places outside the campus. Figure 14 shows the network path we used for the tests. The tests showed that 20,40,80, and 160 users were concurrently sending read requests to the target system 500 times. The Apache Jmeter recorded the response time for each request, which included logging into either the standalone or cloud-based e-book hub system and reading an e-book from the system. The recorded response times served as the measure for the system's performance.

Table 1

The Test Environment

\begin{tabular}{|l|l|}
\hline \multicolumn{2}{|l|}{ Cloud node } \\
\hline Vendor & HP \\
\hline Module & ProLiant DL320 G5p \\
\hline OS & Ubuntu 10.04.3 LTS /OPENSTACK \\
\hline CPU & Intel Xeon X3210(Quad-Core) @ 2.13GHz *1 \\
\hline
\end{tabular}




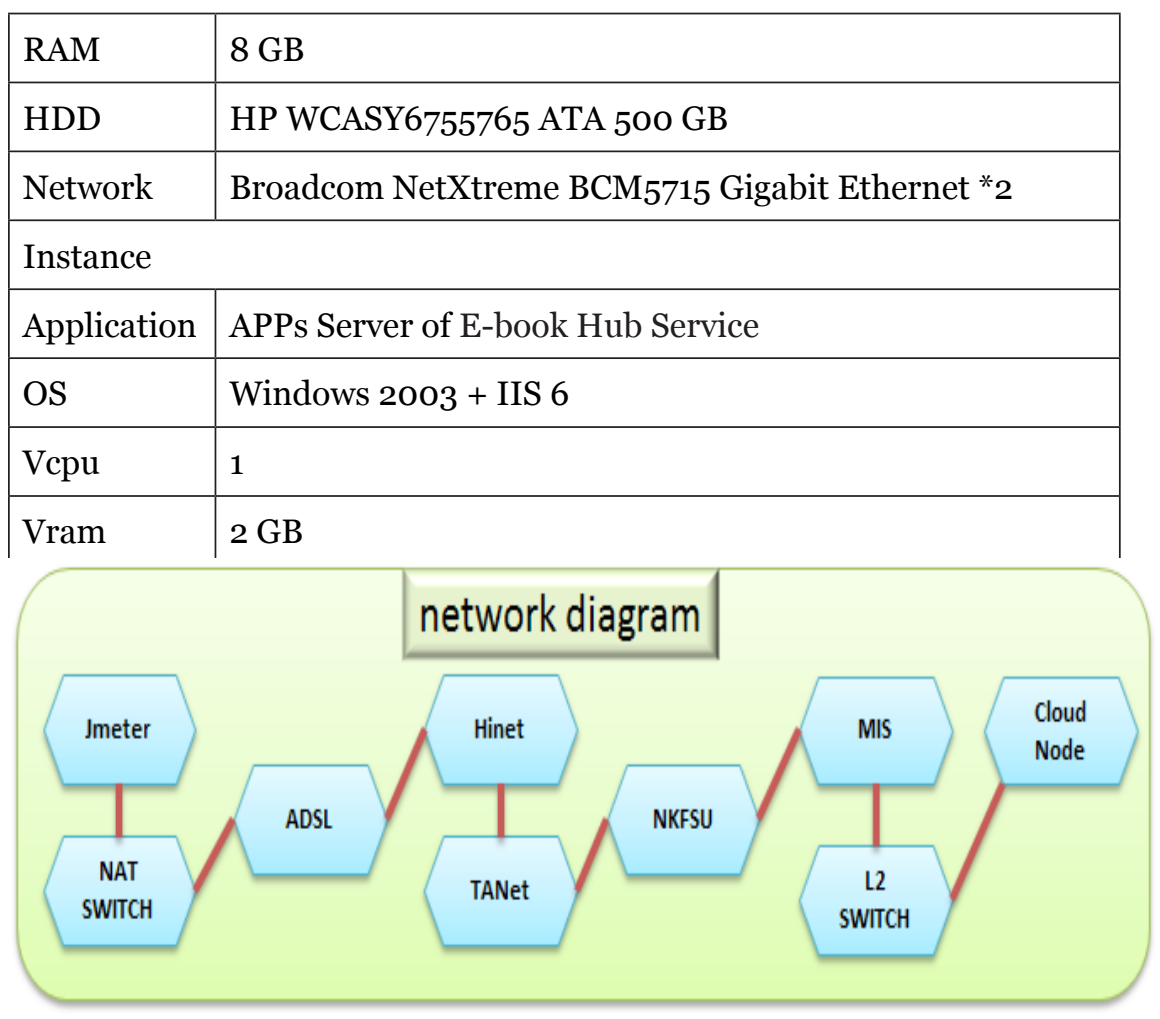

Figure 14. The network path for the test.

We have summarized the simulation results in Table 2. Here are the definitions for the fields in the table.

- $\quad$ Average - The average response time for a set of simulations.

- Median - The median (50\%) response time for a set of simulations.

- $90 \%$ Line - The $90 \%$ quantile response time for a set of simulations. Only $10 \%$ of the simulations had a longer response time than this number.

- $\quad$ Min. - The minimum response time for samples with the same label.

- Max. - The maximum response time for samples with the same label. 
Table 2

Simulation Results, with Response Times in Milliseconds

\begin{tabular}{|c|c|c|c|c|c|c|}
\hline \multicolumn{7}{|c|}{ Single Virtual Machine (stand-alone system) } \\
\hline Label & $\begin{array}{c}\text { \# Sam- } \\
\text { ples }\end{array}$ & Average & $\begin{array}{c}\text { Me- } \\
\text { dian }\end{array}$ & $90 \%$ Line & Min. & Max. \\
\hline 20 users & 10,000 & 403 & 355 & 645 & 45 & 2,696 \\
\hline 40 users & 20,000 & 814 & 744 & 1,298 & 44 & 6,137 \\
\hline 80 users & 40,000 & 1,636 & 1,488 & 2,664 & 45 & 11,765 \\
\hline $\begin{array}{c}160 \text { us- } \\
\text { ers }\end{array}$ & $\begin{array}{c}80,000 \\
\text { HA-Server load balancing (cloud-based system) }\end{array}$ & 3,317 & 2,787 & 5,421 & 52 & 109,505 \\
\hline $\begin{array}{c}\text { Label } \\
\text { ples }\end{array}$ & $\begin{array}{c}\text { \# Sam- } \\
\text { Average }\end{array}$ & $\begin{array}{c}\text { Me- } \\
\text { dian }\end{array}$ & $90 \%$ Line & Min. & Max. \\
\hline 20 users & 10,000 & 417 & 407 & 465 & 139 & 3,528 \\
\hline 40 users & 20,000 & 832 & 796 & 927 & 138 & 4,747 \\
\hline 80 users & 40,000 & 1,666 & 1,556 & 1,898 & 244 & 6,106 \\
\hline $\begin{array}{c}160 \text { us- } \\
\text { ers }\end{array}$ & \begin{tabular}{c}
80,000 \\
\hline
\end{tabular} & 3,354 & 2,900 & 4,898 & 249 & 105,008 \\
\hline
\end{tabular}

The results of our tests show that the cloud-based e-book hub system functioned better than the standalone e-book hub system when the system's load was increased gradually. Compared to the single virtual machine, the $90 \%$ quantile response time of the cloud-based system was shorter in each test environment. According to the $90 \%$ Line statistics, the heavier the load was on the system, the more stable it became.

Figures 15 and 16 present the results of 40,000 requests simulated through 80 users, with 500 read requests per user. As Figure 16 illustrates, the centralization of graphs for the cloud-based system with HA-server load balancing shows that it was more stable than the standalone e-book hub system. In this study, we not only designed a cloudbased e-book hub system, we also implemented it in the OpenStack cloud computing environment. By using cloud computing, the quantity of e-book-provisioning servers could be flexibly increased just in time to meet the sudden demand of a high load. 


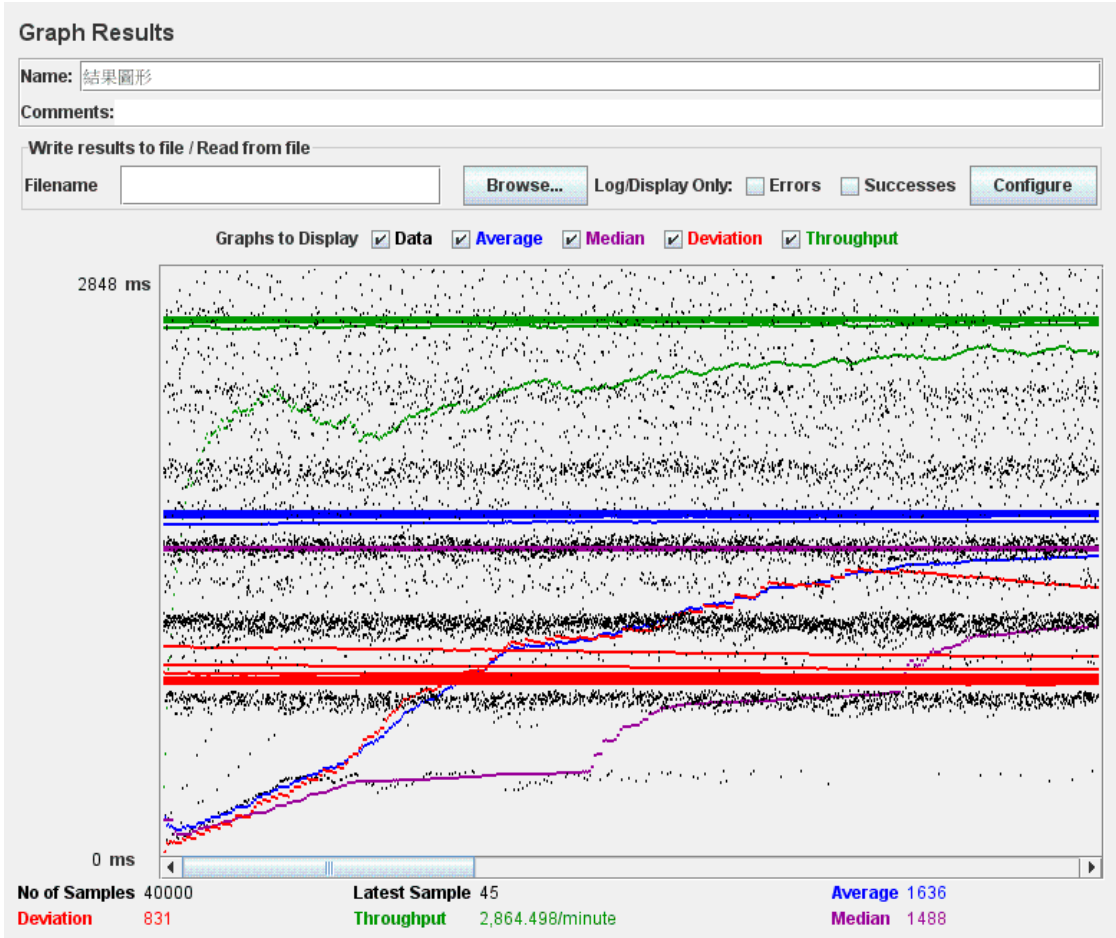

Figure 15. Single virtual machine.

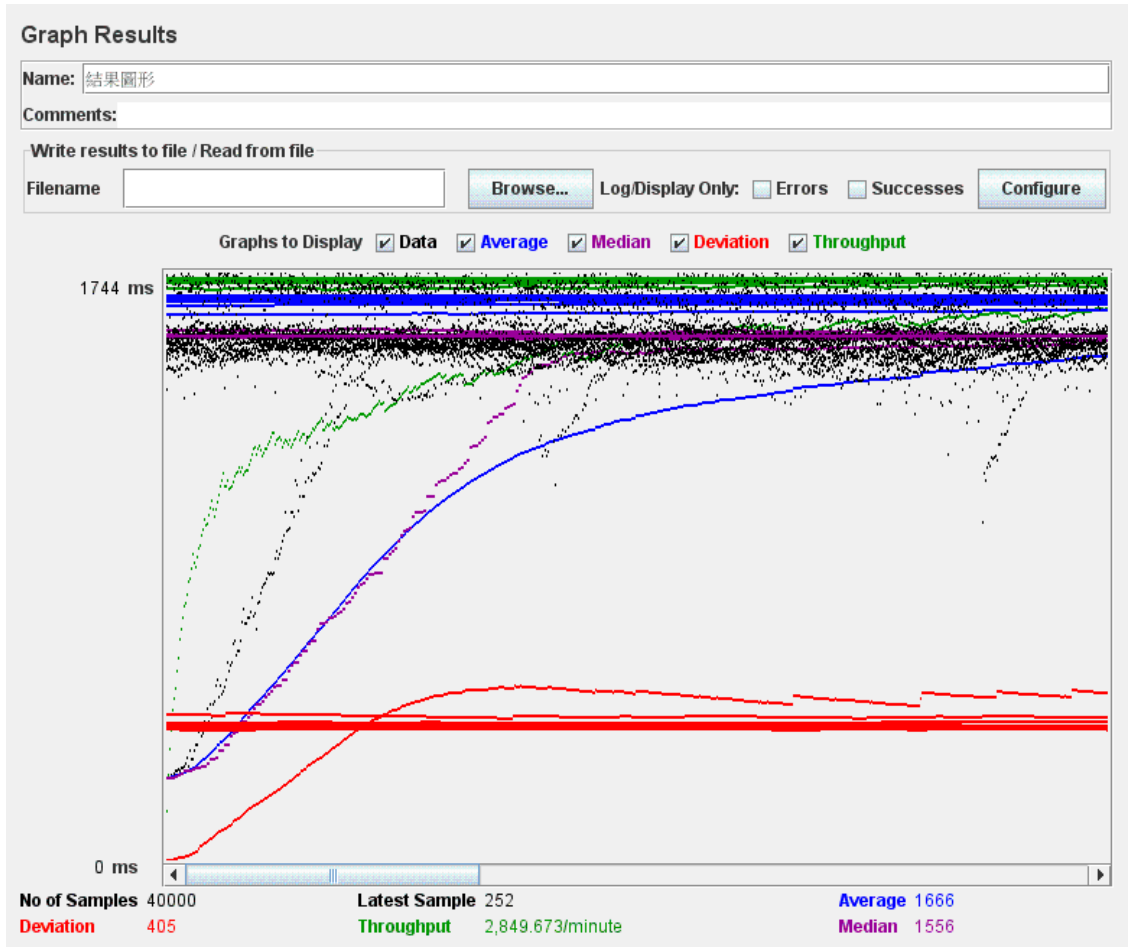

Figure 16. HA-server load balancing structure. 


\section{Conclusions}

In this study, we designed and implemented an e-book hub service on the cloud computing platform to overcome the limitations of storage and computing capabilities inherent in many mobile devices. Our system allowed content providers to easily edit and convert their multimedia e-books and manage the e-book hub service in an unlimited capacity that offered scalable, robust, and safe cloud computing services. Results from this study may help content providers to create a new service model with increased profitability and allow mobile device users to easily access digital content, helping them to achieve the goal of elearning.

We can take a few directions to extend the work we did in this study. So far, the e-book hub system can only operate on Windows servers. We can migrate the developed program to Linux servers to reduce the total cost. Besides, in order to achieve the goal of self-service cloud computing on demand, information from virtual server images should be automatically inserted and converted when it is constructed with the cloud-init component. 


\section{References}

Amazon Kindle (n.d.). In Wikipedia. Retrieved from http://en.wikipedia.org/wiki/Amazon Kindle

Apache Software Foundation (2012). Jmeter [desktop application]. Retrieved from http:// jmeter.apache.org/

Chou, S. C., Stu, J., Lin, Y. T., \& Hsieh, W. T. (2010). Toward computer-based learning: A taxonomy of e-book readers. In Proceedings of the 2nd International Conference on Computer Engineering and Technology (ICCET), Vol. 2 (pp. 431-434).

Haber, L. (2011, August 29). Tablet storage limited, but options abound. TabletPC Review. Retrieved from http://www.tabletpcreview.com/default.asp?newsID=2524\&news $=$ tablet + storage + options + ipad + android + ios

Openstack: The Open Source Cloud Operating System (2012a). About Openstack [Web page] Retrieved from http://www.openstack.org/projects/

Openstack (2012b). Compute [Web page]. Retrieved from http://www.openstack.org/projects/compute/

Openstack (2012c). Image Service [Web page]. Retrieved from http://www.openstack.org/ projects/image-service/

Openstack (2012d). Object Storage [Web page]. Retrieved from http://www.openstack. org/projects/storage/

Rahman, A. S. M. M., Alam, K. M., \& Saddik, A. E. (2011). A prototype haptic eBook system to support immersive remote reading in a smart space. 2011 IEEE International Workshop on Haptic Audio Visual Environments and Games (pp. 124-128).

Sahu, D., Sharma, S., Dubey, V., \& Tripathi, A. (2012). Cloud computing in mobile applications. International Journal of Scientific and Research Publications, 2(8), 1-9.

Schilit B., Price, M., Golovchinsky, G., Tanaka, K., \& Marshall, C. (1999). The reading appliance revolution. Computer, 32(1), 65-73.

Shen, W., \& Ute, K.. (2011). eBooks in the cloud: Desirable features and current challenges for a cloud-based academic eBook infrastructure. Digital Publishing and Mobile Technologies, 15th International Conference on Electronic Publishing (pp. 8086). Retrieved from http://elpub.scix.net/data/works/att/113_elpub2011.content.pdf

SoftBook (n.d.). In Wikipedia. Retrieved from http://en.wikipedia.org/wiki/SoftBook

Sporkin, A. (2012, May 18). US publishers see rapid sales growth worldwide in print and 
e-formats (press release). Retrieved from the AAP, Association of American Publishers Web site: http://www.publishers.org/press/68/

Yee, K. Y., Chia, Y., Tsai, F. S., Tiong, A. W., \& Rajaraman, K. (2011). Cloud-based semantic service-oriented content provisioning architecture for mobile learning. Journal of Internet Services and Information Security, 1(1), 59-69.

\section{Athabasca University $\mathbf{I}$}

(c) (†) 